Check for updates

Cite this: Nanoscale Adv., 2019, 1, 1305

\title{
One-step fabrication of size-controllable nicotine containing core-shell structures
}

\author{
Jeongmin Hong, (D) *ab Rakesh Guduru, ${ }^{c}$ Sakhrat Khizroev ${ }^{c}$ and Long You (D) ${ }^{a}$
}

Received 9th November 2018

Accepted 1st February 2019

DOI: $10.1039 / c 8 n a 00335 a$

rsc.li/nanoscale-advances

We report a one-step synthesis of nicotine-containing nanoparticles by using a size-controllable nanofiltration technique. Nanostructures with polydimethylsiloxane (PDMS) were prepared as a biocompatible well-type polymeric carrier containing a hydrophobic and highly viscous nicotine drug through a novel spontaneous emulsification solvent diffusion method. This approach could be used for efficient dispersion of nicotine in biological systems. Our present results, together with size controllability, pave a way to new types of functional material structures for novel transdermal pharmaceuticals that contain nicotine/cotinine in nanosized structures.

Using conventional nicotine patches to quit smoking may have negative side effects and the effects of nicotine on immune suppression are widely recognized. ${ }^{1}$ Little is known about the effect of the size of administered nicotine particles on the immunosuppression. The use of nicotine-containing nanoparticles is a possible way to prevent immunosuppression, and it is important to carry out comparative drug release studies on size-controllable nicotine/cotinine-containing biocompatible nanostructures.

Drug delivery systems conventionally rely on pills, drops, ointments, injections, and other routes of administration. ${ }^{2}$ Recently, a number of novel drug delivery approaches have been developed, including drug modification by chemical approaches, such as drug entrapment in micro-vesicles that can be injected into the bloodstream, or drug entrapment within pumps or polymeric materials that are placed in desired bodily compartments (e.g., prolonged action or continual-release pills beneath the skin). ${ }^{3}$

To administer nicotine, which is a highly viscous fluid, the use of transdermal or edible protective polymer composites is

${ }^{a}$ School of Optical and Electronic Information, Huazhong University of Science and Technology, Wuhan 430074, PR China. E-mail: jehong@hust.edu.cn

${ }^{b}$ Research and Development Division, JS Nanotechnologies LLC, San Jose, California 95134, USA

'Electrical and Computer Engineering, University of Miami, Coral Gables, Florida 33159, USA required. Due to its high viscosity, control of the administration time of nicotine is very important to ensure successful timedependent delivery. Thus, new methods for the fabrication of highly dispersible nanocomposites, which contain drugs of high viscosity, should be investigated. ${ }^{4}$

We have found a novel approach that could be used to fabricate nicotine-containing particles that range in size from thousands to hundreds of nanometers. ${ }^{5}$ This approach results in liquid nanoparticles that are characterized by a particularly small size of approximately 10-500 $\mathrm{nm}$ (depending on the concentrations of individual components employed). According to the chemical synthesis process, on the surface of each nanoparticle is a well-type core structure, which contains physiologically acceptable oils together with the dissolved nicotine. Because of their composition, on penetration into the horny layer of the epidermis, nanoparticles produced in this manner are expected to freely integrate into the lipid bilayers of skin cells, and from there, active nicotine can permeate in a very controlled manner into the deeper living skin layers. The size of the nanoparticles is controlled by the proportion of water in the dispersion from approximately 50 to over $200 \mathrm{~nm}$. The mechanism of nanosphere formation was verified by the physiochemical properties of the nanoparticles, such as particle diameter, surface topography, and drug content.

In this study, we fabricated nicotine-containing PDMS/PVA nanocomposites by a one-step fabrication procedure. There are 5 major procedures which could be merged into a single step: stock solution, emulsification, solidification, filtration, and characterization. First, from the stock solution, we used polydimethylsiloxane (PDMS), nicotine, acetone, and chloroform. ${ }^{6}$ Initially, $15 \mathrm{ml}$ of chloroform was mixed with $25 \mathrm{ml}$ of acetone. Then, $120 \mathrm{mg}$ of PDMS and $24 \mathrm{mg}$ of nicotine were mixed by agitation and dissolved in the stock solution. The structure after dissolution is shown in Fig. 1.

For the second procedure, i.e., emulsification, we prepared an aqueous PVA solution by mixing $2 \mathrm{~g}$ of PVA in $50 \mathrm{ml}$ of deionized water $(4.0 \%$ of PVA, w/v). The solution was stirred using a magnetic bar at $50{ }^{\circ} \mathrm{C}$ for 36 to $48 \mathrm{~h}$ followed by further 


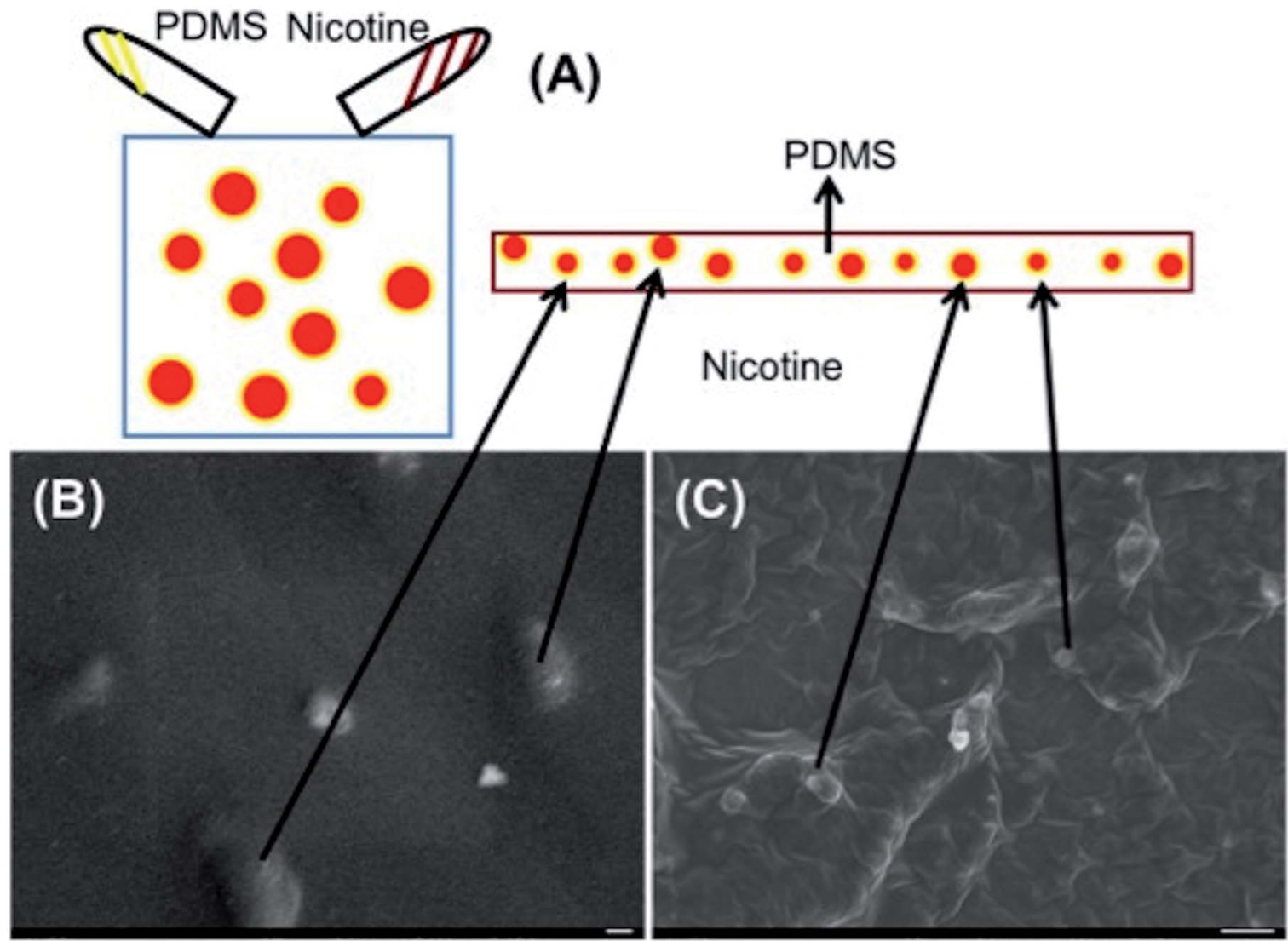

Fig. 1 (A) Schematic diagram of the procedure for fabricating a stock solution containing PDMS and nicotine. (B) SEM image of nicotinecontaining PDMS composites. (C) SEM image of small-sized nicotine-containing PDMS composites. Scale bar: $100 \mathrm{~nm}$.

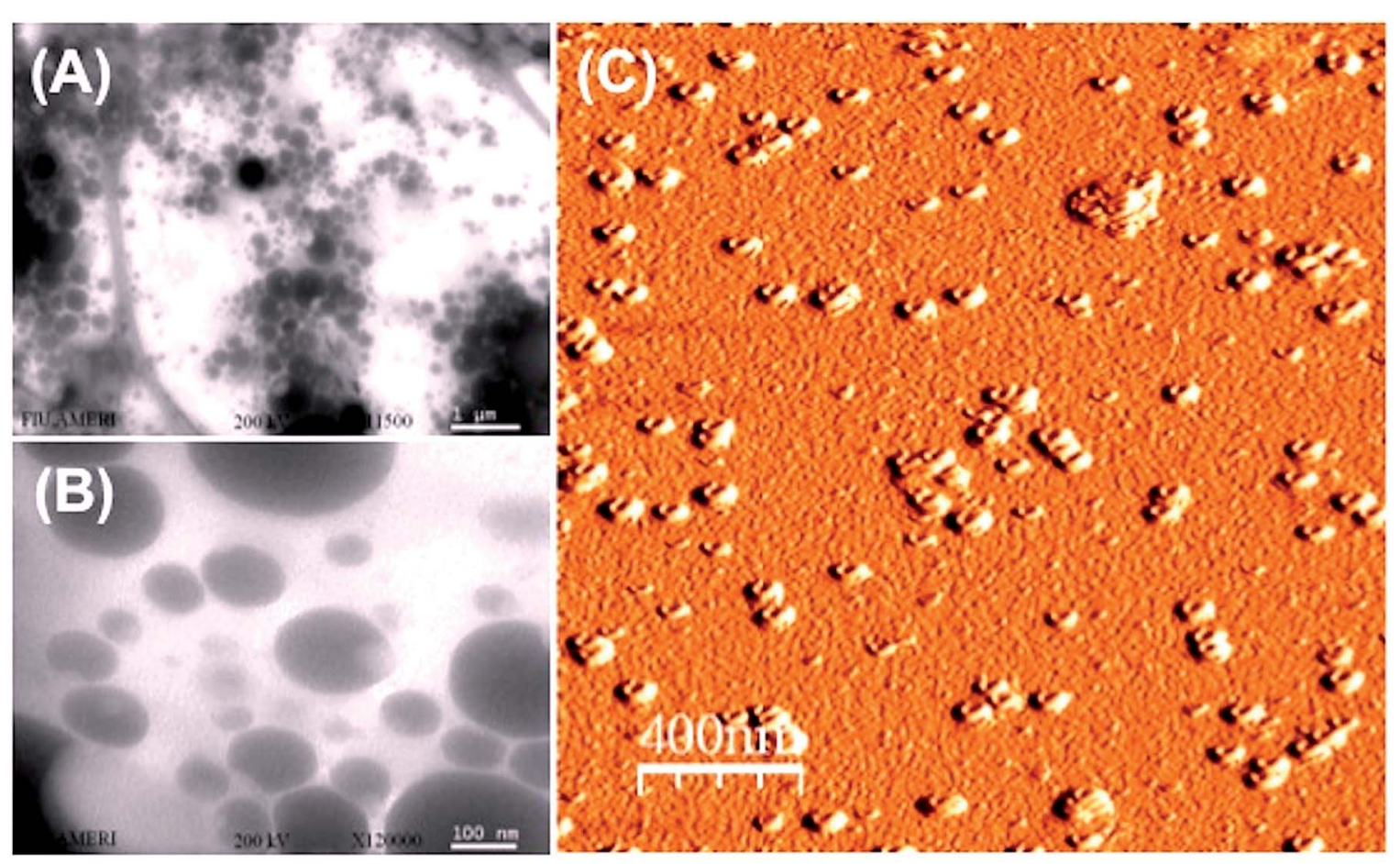

Fig. 2 (A) TEM image of larger-sized nicotine-containing PDMS/PVA composites. (B) TEM image of smaller-sized composites. (C) AFM image of nicotine-containing well-type PDMS/PVA nanocomposites. 
stirring at room temperature for $24 \mathrm{~h}$ or until PVA was completely dissolved. The PVA solution was then stirred at $3000 \mathrm{rpm}$ with a mechanical stirrer for several minutes, and the resulting solution was emulsified into nanodroplets in $50 \mathrm{ml}$ of aqueous PVA solution $(4.0 \% \mathrm{w} / \mathrm{v})$ under stirring at $3000 \mathrm{rpm}$ using a mechanical stirrer for about $3 \mathrm{~h}$.

For the solidification procedure, we mixed the resulting solution using a magnetic stirrer for $12 \mathrm{~h}$ and the core-shell
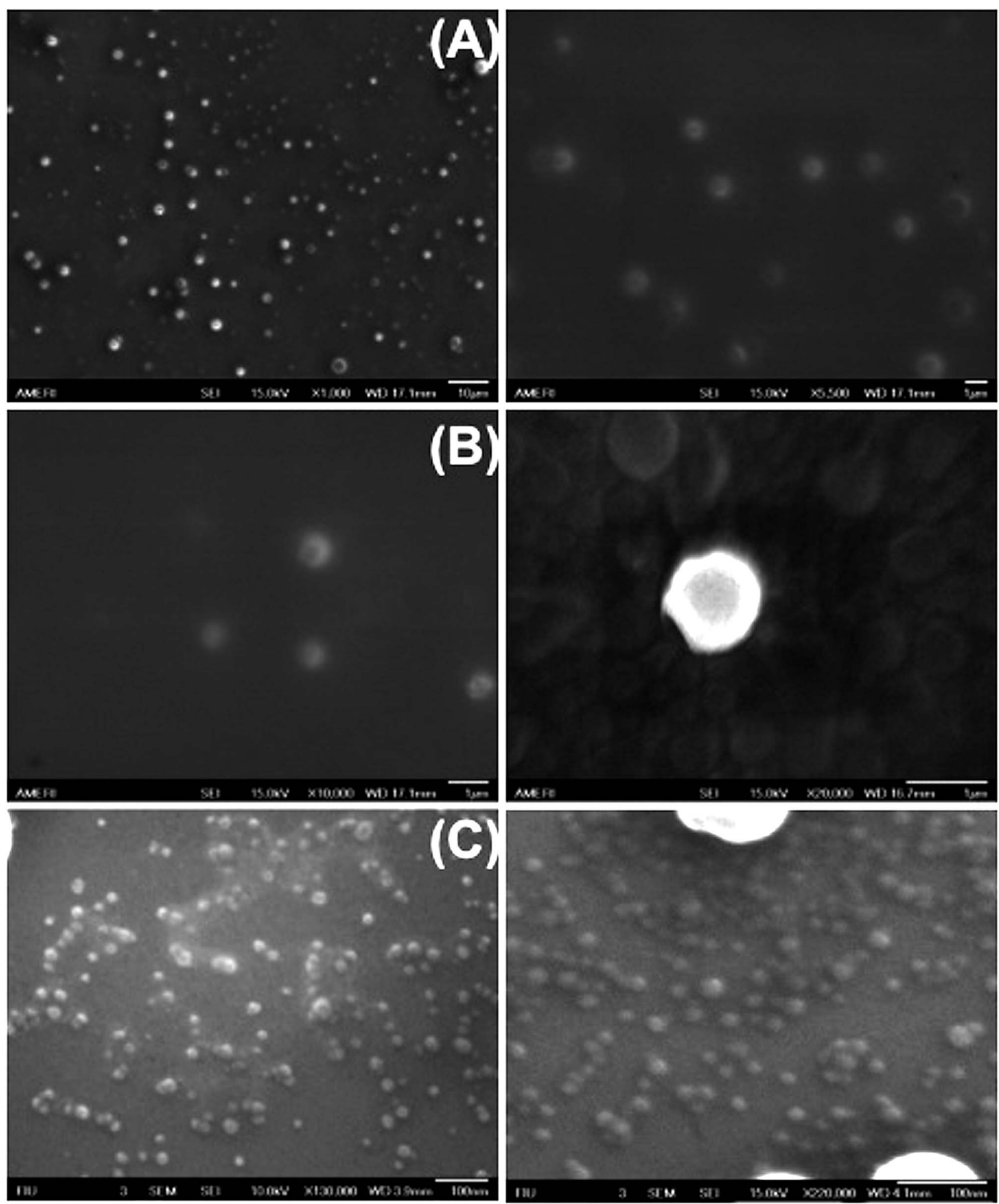

Fig. 3 (A) SEM images of the nicotine-containing core-shell structure before filtration. (B) SEM images of the nicotine-containing core-shell structure after filtration. (C) SEM image of the nicotine-containing core-shell structure after the finest filtration. 
structures produced are shown in Fig. 2. Well-type core-shell structures could be clearly observed. The dispersed particles were then filtered through a fine filter $(\sim 5 \mu \mathrm{m})$ to remove largesized particles. The particles were then centrifuged (13 $000 \mathrm{rpm}$ for approximately $2 \mathrm{~h}$ ), the supernatant was removed, and particles were slightly diluted with deionized water. The resulting solution was again filtered through $100 \mathrm{~nm}$ and $10 \mathrm{~nm}$ sized anodic alumina membranes, respectively and then centrifuged at $13000 \mathrm{rpm}$ for $3 \mathrm{~h}$. Particles were obtained by removing the supernatant at each filtration step using two different types of membranes $(100 \mathrm{~nm}$ and $10 \mathrm{~nm}$ of membrane diameters), respectively.
The particles were diluted with deionized water in order to prepare the samples for characterization by scanning electron microscopy (SEM), transmission electron microscopy (TEM), and atomic force microscopy (AFM). TEM was performed using a Philips CM-200. SEM was performed using a JEOL 6330F Field Emission Scanning Electron Microscope (FE-SEM). Energy dispersive X-ray spectroscopy (EDS) was performed using a Thermo EDS system mounted on a JEOL 6330F system. Scanning probe microscopy was carried out by non-contact mode AFM.

One of the main objectives of the study is to reduce the size of the particles. A spontaneous emulsification method for the formation of PDMS composite nanoparticles made it possible to drastically reduce particle size. Due to its various uses as
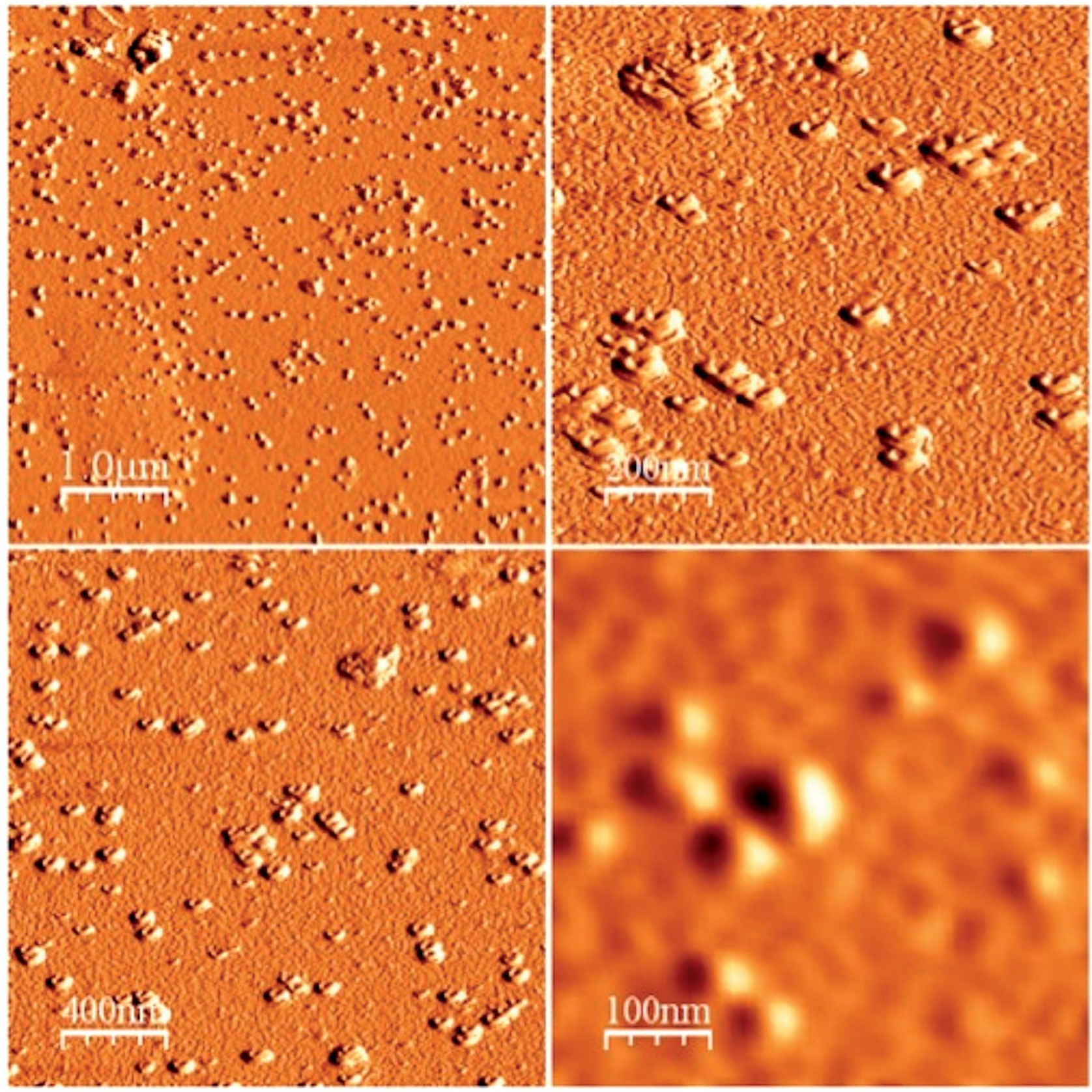

Fig. 4 AFM images of the nicotine-containing core-shell structure after filtration. 
a stable structure for electronics and medicine, such as MEMS (NEMS) and Bio-MEMS (Bio-NEMS), PDMS could be used to ensure a biocompatible and stable structure after solidification. ${ }^{7}$ Nanofiltration ensures size-controllability, as determined by the size of the meshes used. Because of agitation from rigorous stirring and differences in the viscosity of the materials, drug-containing composites formed as a gel structure.

Biodegradable polymers are well-known structures for drug delivery applications, but there are some difficulties in controlling the flow rate, and particle size is important.
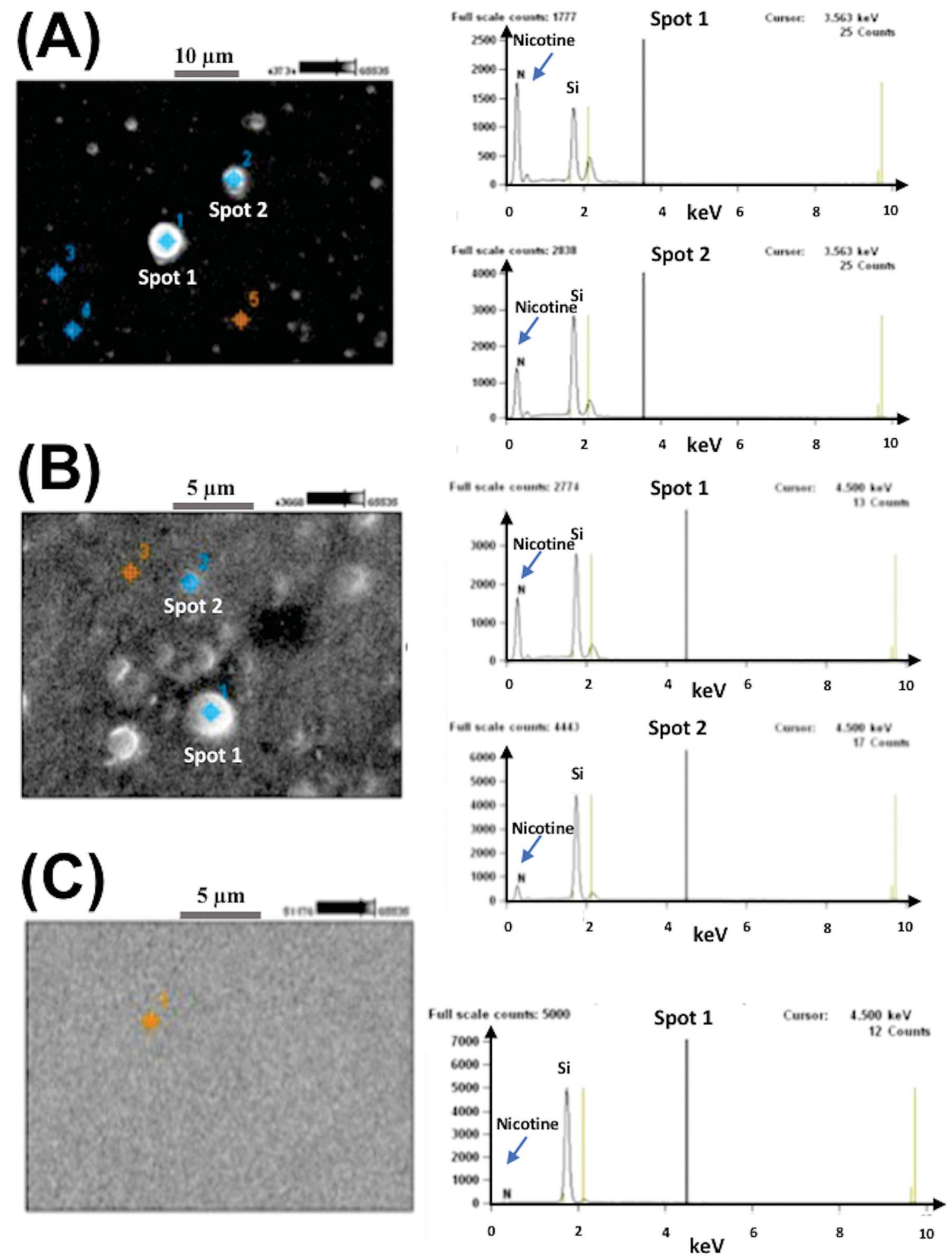

Fig. 5 Energy Dispersive X-ray Spectroscopy (EDS) of the nicotine-containing core-shell structure. (A) High concentration of nicotine containing nanoparticles. (B) Low concentration of nicotine containing nanoparticles. (C) Silicon substrate for reference. 
However, PDMS could ensure that well-type structures could be used to deliver drugs to biological systems with stable flow rates. According to the chemical synthesis process, the surface of each nanoparticle is composed of the well-type core of the nanoparticle, which contains physiologically acceptable solvents. ${ }^{8}$ On account of their composition, upon penetration into the horny layer of the epidermis the nanoparticles produced in this manner are expected to freely integrate into the lipid bilayers of the skin barrier, and from there release active nicotine in a very controlled manner into the deeper living skin layers through transdermal permeation.

Drugs containing PDMS can form as composites, mainly because of extremely different viscosities (microemulsion). One advantage of this is that once the nicotine-containing composites form, they diffuse into the body at an extremely stable flow rate. ${ }^{9-11}$ Schematic procedures for preparing the stock solution containing nicotine and PDMS are shown in Fig. 1(A). After the process was complete, we could observe the composites containing PDMS and nicotine as seen in Fig. 1(B) and $(\mathrm{C})$.

In addition, polyvinyl alcohol (PVA) is a beneficial compound because it has excellent emulsifying properties. These properties are dependent on humidity; in other words, with higher humidity more water is absorbed. The water, which acts as a plasticizer, reduces its tensile strength, but increases its elongation and tear strength. PVA is an atactic material but exhibits crystallinity as the hydroxyl groups are small enough to fit into the lattice without disrupting it. With PVA, a water-
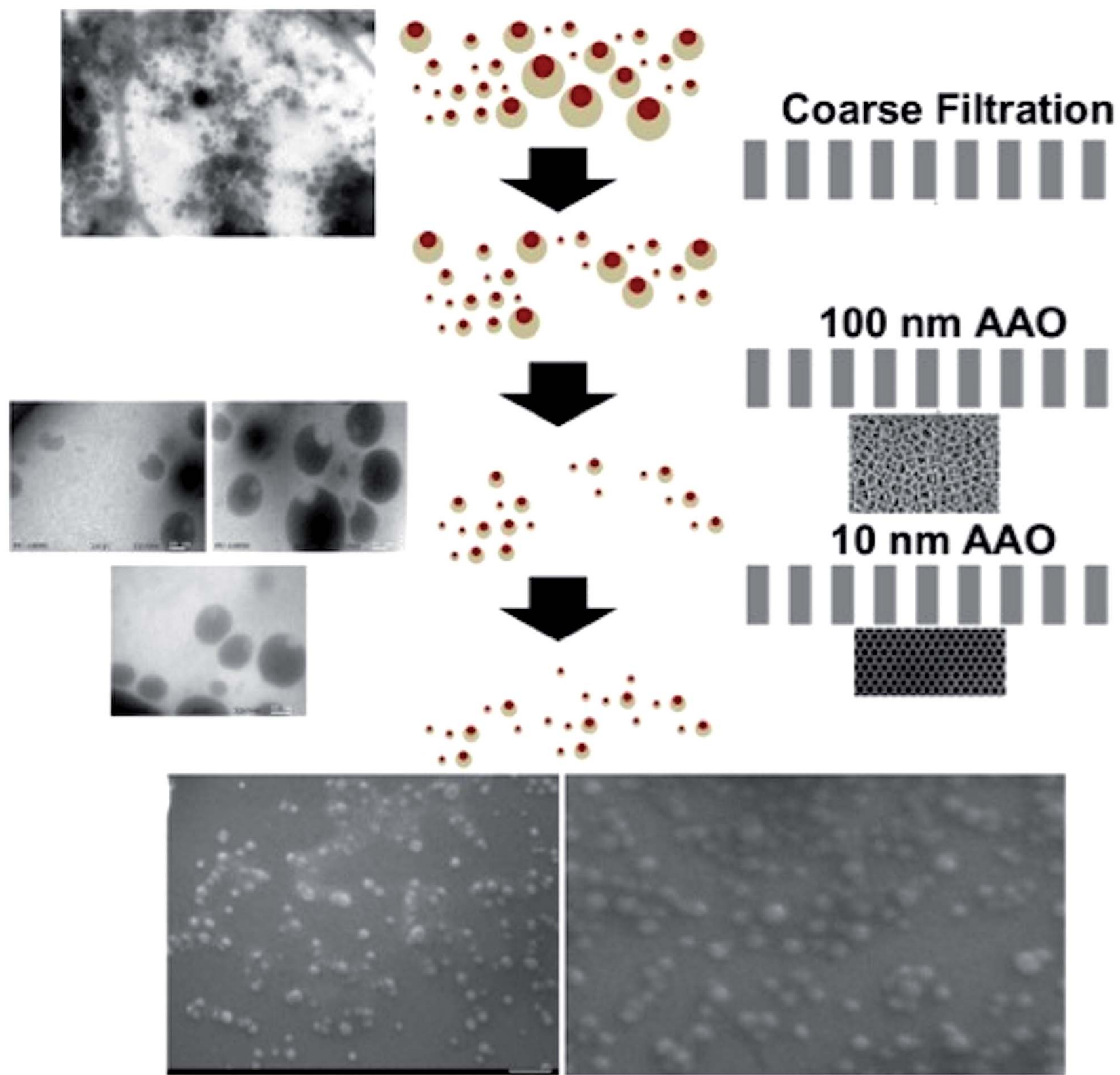

Fig. 6 Procedure for the formation of PDMS/nicotine wells in PVA core-shell structures and filtration. Schematic description of the filtration procedure using 2 different sized AAO membranes (10 $\mathrm{nm}$ and $100 \mathrm{~nm}$, respectively). 
soluble synthetic polymer, well-type formation occurred because of agitation by rigorous mechanical stirring. ${ }^{12}$ The welltype core-shell structures were formed by microemulsion and solidification approaches, as could be confirmed from Fig. 2(A), (B), and (C): TEM image of larger-sized nicotine-containing PDMS/PVA composites, TEM image of smaller-sized

\section{(1) Stock Solution}
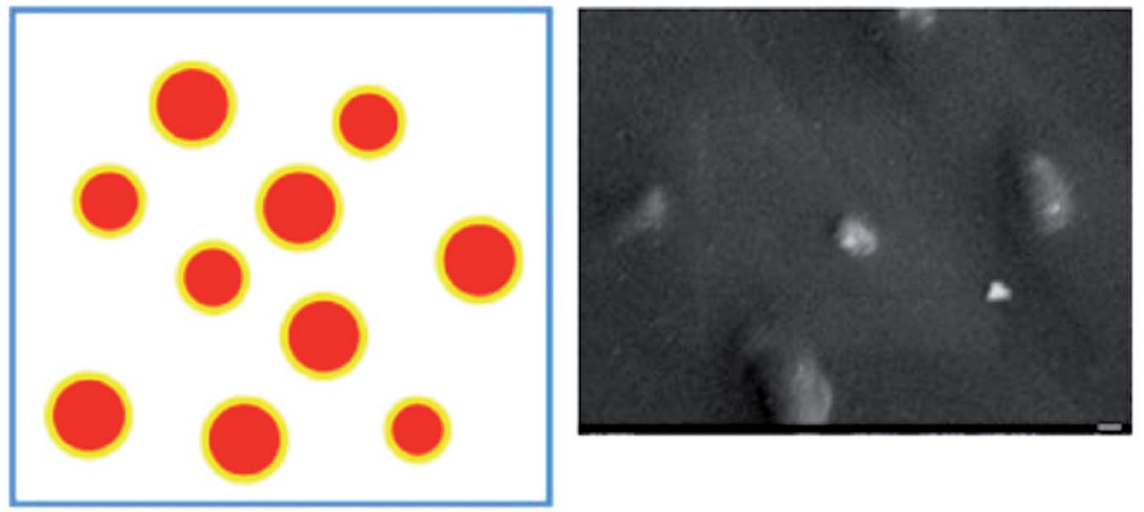

(2) Emulsification

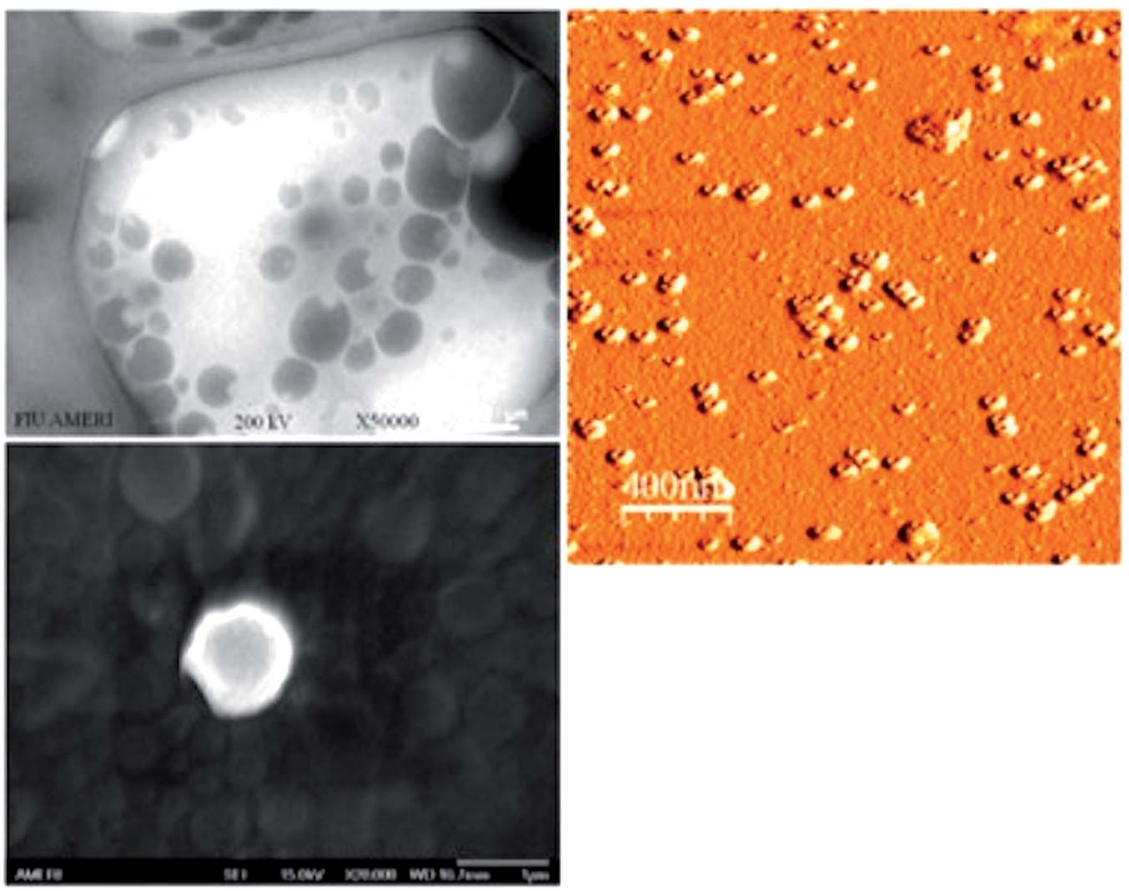

(3) Filtration

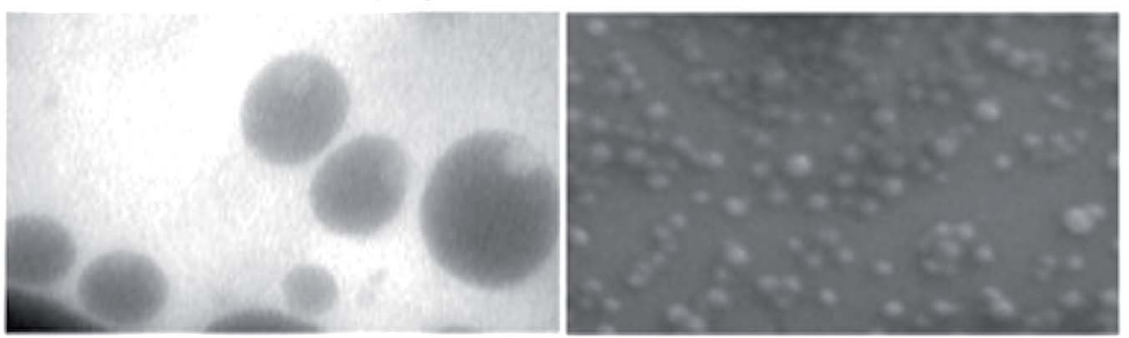

Fig. 7 Procedure for one-step fabrication of nicotine-containing PDMS/PVA nanocomposites. (1) Stock solution, (2) emulsification and solidification, and (3) filtration, respectively. 
composites, and AFM image of nicotine-containing well-type PDMS/PVA nanocomposites, respectively. TEM studies demonstrate the perfect structure of nicotine-containing core-shell nanoparticles and nicotine-containing PDMS wells were found inside the hydrophobic PVA structure.

To investigate the size-controllable spontaneous emulsification solvent diffusion method for the formation of PDMS nanoparticles, we applied nanofiltration, which can control particle size depending on the size of the membrane. We could observe the degrees of filtration at each step with reference to Fig. 3(A) SEM images of nicotine-containing core-shell structure before filtration, Fig. 3(B) SEM images of nicotine-containing core-shell structure after filtration, and Fig. 3(C) SEM image of nicotinecontaining core-shell structure after the finest filtration, respectively. As shown in Fig. 4, we could observe well-type structures after filtration by AFM measurement.

The EDS pattern demonstrated the presence of nicotine in core-shell structures inside hydrophobic vehicles. Nitrogen composition is a signature of the percentage of nicotine present, and from the composition of nitrogen, the percentage of particles can be determined. These types of biocompatible materials are excellent for delivery systems because of their stable structures for controlling flow rates. Using EDS, we could confirm that the well-type core-shell structures contained nicotine as shown in Fig. $5 .^{13}$

Systematic nanofiltration demonstrates the extremely accurate size controllability of the nanodots by controlling the flow rate of the solutions as shown in Fig. 6. We could observe systematic filtration based on the size of the filtration membrane. A schematic description of the filtration procedure using 2 different sized AAO membranes $(10 \mathrm{~nm}$ and $100 \mathrm{~nm}$, respectively) is shown in Fig. 6.

In summary, we describe a one-step fabrication procedure for nicotine-containing PDMS/PVA composites. With the stock solution used, PVA emulsification and solidification, and filtration, we could fabricate size-controllable nicotinecontaining PDMS/PVA composites to ensure extremely stable flow rates. The complete procedure employed in this study is shown in Fig. 7.

\section{Conclusions}

We find that an optimized agitation and emulsification make nanoparticles stable for drug delivery. After further solidification, nanoparticles formed perfect core-shell structures. Size controllability could be optimized by nanofiltration from $10 \mathrm{~nm}$ to $100 \mathrm{~nm}$ when using a bulk-fabricated nicotine-containing well-type core-shell structure. By using our novel approach, it is possible to fabricate nicotine-containing particles that vary in size from several nanometers to hundreds of nanometers. This results in liquid nanoparticles that are characterized by a particularly small size of approximately 10-500 nm (depending on the concentrations of the individual components). The nanocomposites produced by this approach can be expected to be useful as carriers of physiologically active agents, such as transdermal penetration into the horny layer of the epidermis in a very controlled manner, and from there into the deeper living skin layers at an extremely stable flow rate. Therefore, their discrete sub-nanometer structure is attractive for applications to nicotine administration using transdermal nicotine patches.

\section{Conflicts of interest}

There are no conflicts to declare.

\section{Acknowledgements}

This work was supported by the National Natural Science Foundation of China under Award number 61674062. The work was also supported by the U. S. Department of Energy, Office of Basic Energy Sciences, Division of Materials Sciences and Engineering under Contract No. DE-AC02-05CH11231.

\section{Notes and references}

1 M. Sopori, Effects of cigarette smoke on the immune system, Nat. Rev. Immunol., 2002, 2, 372-377.

2 R. H. Muller, K. Mader and S. Gohia, Solid lipid nanoparticles (SLN) for controlled drug delivery - a review of the state of the art, Eur. J. Pharm. Biopharm., 2000, 50, 161-177.

3 R. Lagner, Drug delivery and targeting, Nature, 1998, 392, 510.

4 L. Y. Qiu and Y. H. Bae, Polymer architecture and drug delivery, Pharm. Res., 2006, 23, 1.

5 H. Samber, K. Hoshina, R. Moaddel, I. W. Wainer and J. Haginaka, Uniformly-sized, molecularly imprinted polymers for nicotine by precipitation polymerization, $J$. Chromatogr. A, 2006, 1134, 1.

6 T. Niwa, H. Takeuchi, T. Hino, N. Kunou and Y. Kawashima, Preparations of biodegradable nanospheres of water-soluble and insoluble drugs with D,L-lactide/glycolide copolymer by a novel spontaneous emulsification solvent diffusion method, and the drug release behavior, J. Controlled Release, 1993, 25, 89-98.

7 B. J. Blaiszik, S. L. B. Kramer, S. C. Olugebefola, J. S. Moore, N. R. Sottos and S. R. White, Self-Healing polymers and composites, Annu. Rev. Mater. Res., 2010, 40, 179-211.

8 D. E. Mager, V. Mody, C. Xu, A. Forrest, W. G. Lesniak, S. S. Nigavekar, M. T. Kariapper, L. Minc, M. K. Khan and L. P. Balogh, Physiologically based pharmacokinetic model for composite nanodevices: Effect of charge and size on in vivo disposition, Pharm. Res., 2012, 29, 2534-2542.

9 T. Trantidou, Y. Elani, E. Parsons and O. Ces, Hydrophilic surface medication of PDMS for droplet microfluidics using a simple, quick and robust method via PVA deposition, Microsyst. Nanoeng., 2017, 3, 16091.

10 C.-H. Choi, H. Lee and D. A. Weitz, Rapid patterning of PDMS microfluidic device wettability using syringevacuum-induced segmented flow in nonplanar geometry, ACS Appl. Mater. Interfaces, 2018, 10, 3170-3174.

11 A. Gokaltun, M. L. Yarmush, A. Asatekin and O. Berk Usta, Recent advances in nonbiofouling PDMS surface 
modification strategies applicable to microfluidic technology, Technology, 2017, 5, 1-12.

12 G. Schmidt and M. M. Malwitz, Properties of polymernanoparticle composites, Curr. Opin. Colloid Interface Sci., 2003, 8, 103-108.
13 J. Hong, E. Stefanescu, P. Liang, N. Joshi, S. Xue, D. Litvinov and S. Khizroev, Carbon nanotube based 3-D matrix for enabling three dimensional nano-magneto-electronics, PLoS One, 2012, 7, e40554. 